\title{
Hyperbaric Oxygen Treatment for Diabetic Retinopathy and Neuropathy in a Streptozotocin Induced Diabetic Rat Model
}

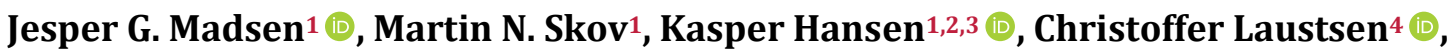 \\ Niels Ejskjær ${ }^{5,6}$ (), Henning Andersen 7 (), Michael Pedersen 1 (i) \\ ${ }^{1}$ Department of Clinical Medicine, Comparative Medicine Lab, Aarhus University, Aarhus, Denmark; \\ ${ }^{2}$ Zoophysiology, Department of Biology, Aarhus University, Aarhus C, Denmark; ${ }^{3}$ Department of Forensic Medicine, \\ Aarhus University, Aarhus N, Denmark; ${ }^{4}$ Department of Clinical Medicine, The MR Research Centre, Aarhus \\ University, Aarhus, Denmark; ${ }^{5}$ Department of Clinical Medicine, Aalborg University, Aalborg, Denmark; ${ }^{6}$ Steno \\ Diabetes Center North Denmark, Aalborg University Hospital, Aalborg, Denmark; ${ }^{7}$ Department of Neurology, \\ Aarhus University Hospital, Aarhus, Denmark
}

Correspondence to: Jesper G. Madsen, jesper.madsen@clin.au.dk

Keywords: Diabetic Neuropathy, Diabetic Retinopathy, Hyperbaric Oxygen Therapy

Received: October 30, $2021 \quad$ Accepted: December 13, $2021 \quad$ Published: December 16, 2021

Copyright $\odot 2021$ by author(s) and Scientific Research Publishing Inc.

This work is licensed under the Creative Commons Attribution International License (CC BY 4.0).

http://creativecommons.org/licenses/by/4.0/

\section{(c) (i) Open Access}

\section{ABSTRACT}

Aim: Diabetes Mellitus is a global public health challenge with major and potentially devastating complications, and concomitant complications include retinopathy and neuropathy due to hypoxia and microvascular dysfunction. In this study, we investigated the effect of hyperbaric oxygen therapy as a method to transiently improve tissue oxygenation on diabetic retinopathy and neuropathy in a streptozotocin induced type-1 diabetic rat model (Wistar). Methods: Streptozotocin induced type-1 diabetic rats received 10 sessions of 2-h hyperbaric oxygen exposures $\left(\mathrm{pO}_{2}=309 \mathrm{kPa}\right)$ over 2 weeks. Animals were exposed to light stimuli to produce light evoked potentials to estimate the effect of oxygen treatment on diabetic retinopathy. Sciatic nerves were exposed and stimulated to produce muscle evoked potential, which were recorded in the muscles of the foot and subsequently used to evaluate the effect of oxygen treatment on diabetic neuropathy. Results: We found significantly shorter light evoked potential latency and increased amplitude in hyperbaric oxygen treated animals. No change was found in nerve conduction. Conclusions: This study showed that hyperbaric oxygen therapy is a potentially effective treatment for diabetic retinopathy, improving both latency and amplitude of light evoked potentials. 


\section{INTRODUCTION}

Diabetes Mellitus (DM) is a fast growing health problem in the developed world with an estimated 422 million adults living with DM in 2014 [1]. Two of the most frequent complications associated with $\mathrm{DM}$ are diabetic retinopathy (DR) and diabetic neuropathy (DN); in fact, one in 12 people with $\mathrm{DM}$ in the US is estimated to have advanced vision degrading retinopathy [2], while the prevalence of neuropathy is approximately $30 \%$ [3]. The quality of life is generally low for those suffering from these diseases, including development of blindness, sensation loss, neuropathic pain, foot ulcers and amputation of extremities.

Common for DR and DN is reduced capillary function in the blood vessels, resulting in hypoxic conditions $[4,5]$. Over time, hyperglycemia can cause basement membrane thickening in capillaries supplying peripheral nerves, with demyelination and loss of fibers as consequence of disrupted blood flow and poor oxygen extraction [6-11]. In the retina, reduced blood flow causes increased angiogenesis [12, 13], leading to vitreous hemorrhage, retinal detachment and neovascular glaucoma which ultimately result in loss of visual acuity.

Thus, the aim of this study was to investigate if hyperbaric oxygen therapy (HBOT) is able to halt the progression towards DR and DN, in streptozotocin (STZ) induced type-1 diabetic rats. The STZ induced diabetic rat model has been used extensively in studies of both DR [14-16] and DN [17-19].

HBOT for DR and DN has received little attention. Most research concerning HBOT has been performed on diabetic foot ulcers $[20,21]$. However, there is evidence that HBOT may improve peripheral nerve function [22] in DM. DM has also been linked to delayed onset of light evoked potentials (LEP) [23, 24]. In the present study, we investigated the effect of HBOT on signal amplitudes of muscles stimulated by peripheral motor neurons along with nerve conduction velocity. Furthermore, we examined the effect of HBOT on LEP amplitudes and onset times. With these electrophysiological measurements, we seek to determine whether HBOT may be a viable therapeutic option in diabetes.

We hypothesized that transient increases in tissue oxygen tension caused by HBOT may alleviate symptoms caused by DR and DN, specifically, higher latency and decreased amplitude of LEPs for DR and decreased conduction velocity and decreased MEPs for DN.

\section{MATERIALS AND METHODS}

We included 36 female Wistar rats (Janvier, France) with a weight of $250-400 \mathrm{~g}$ and an age of 12 weeks. The animals were divided into 4 groups of 9 animals per group:

+HBOT/+Diabetes, +HBOT/-Diabetes, $-\mathrm{HBOT} /+$ Diabetes and -HBOT/-Diabetes. Diabetes was induced with STZ (Sigma Aldrich), $45 \mathrm{mg} / \mathrm{kg}$ in cold $\left(2^{\circ} \mathrm{C}-3^{\circ} \mathrm{C}\right) 0.01 \mathrm{M} \mathrm{pH} 4.5$ sodium citrate buffer, via the tail vein after an overnight fast [25]. Animals were confirmed diabetic $48 \mathrm{~h}$ after induction. Induced animals had ad libitum access to food and water. HBOT was initiated two weeks following STZ-induction as previous studies have shown that such duration is sufficient to produce effects of DM in peripheral nerves $[19,26]$. Rats were anesthetized using intraperitoneal administration of ketamine $(100 \mathrm{mg} / \mathrm{kg})$ and xylazine $(10 \mathrm{mg} / \mathrm{kg})$ [27] before onset of electrophysiology experiments.

The applied procedures and HBOT-protocol was similar to a previous STZ induced diabetic animal study [28]. In short, HBOT consisted of 10 sessions of pressurization with $100 \%$ oxygen to $309 \mathrm{kPa}(3 \mathrm{~atm})$ with 5 pressurization sessions per week. Animals were placed in a $75 \mathrm{~L}$ custom made pressure chamber, 9 animals per session. Animals were kept at $309 \mathrm{kPa}$ for $2 \mathrm{~h}$. Compression and decompression rates were 30 $\mathrm{kPa} / \mathrm{min} . \mathrm{CO}_{2}$ removal was achieved using a $\mathrm{CO}_{2}$ scrubber and by maintaining a continuous $(15 \mathrm{~L} / \mathrm{min})$ flow of oxygen whenever animals resided the pressure chamber. Non-HBOT animal groups (-HBOT/ + Diabetes and -HBOT/-Diabetes) were given sham sessions of equal duration without pressurization inside the pressure chamber.

LEPs were evoked by $30 \mathrm{~ms}$ white light flashes from a COB-POWER-LED (5 W 450 - 550 lumen). Power (10 V, $5 \mathrm{~mA})$ and timing for the LED were supplied by a signal generator (model A310; World Precision Instruments (WPI), Sarasota FL, USA) through a signal isolator (WPI A365). The signal generator was controlled by a PC executing a custom build script in LabView (2013 v. 13-0f2) software, via a digi- 
tal-to-analog converter (model USB 6152; National Instruments (NI), Austin TX, USA). Light stimulation occurred inside a dark box where two stainless-steel needle electrodes were placed subcutaneously, one pole above the eye, the other above the occipital lobe.

Muscle evoked potentials (MEPs) were evoked by stainless-steel electrodes fastened to the exposed sciatic nerves. The nerves were stimulated with pulses of $10 \mathrm{~V}$ and $3 \mathrm{~ms}$ duration using the same stimulation setup as described for LEPs. Stainless-steel electrodes were placed subcutaneously in the flexor digitorum brevis muscle of the foot, and they allowed recording the resulting MEPs.

All recorded potentials were amplified via a preamplifier (model P50; Grass, W. Warwick, RI, USA) set to 10x amplification for MEPs and 100x amplification for LEPs and bandpass filter settings between 0.1 $\mathrm{Hz}$ and $3 \mathrm{kHz}$. Sample rate was $20 \mathrm{kHz}$. All electrophysiological experiments were conducted inside a Faraday cage. Recorded data were stored on a PC via the digital-to-analog converter (see Figure 1 for detailed description of the electrophysiological setup).

LEPs were calculated from the peak-peak amplitude of the evoked potentials and onset time of stimulus response post-stimulation. Peak-peak amplitudes were calculated by subtracting the lowest record voltage of the LEPs from the highest recorded. MEPs were quantified using root mean square (RMS) values of the evoked potentials. RMS was utilized for MEPs as these evoked potentials consisted of complex wave forms containing several peaks, whereas LEPs contained only one peak. Nerve-muscle conduction speed was calculated by dividing the length between stimulation and recording electrodes with the time of onset of the MEPs.

\subsection{Histology}

After euthanasia, nervous tissue was removed immediately after stimulations in each animal from the site of stimulation and preserved in $9 \%$ formalin solution. Nerve sections were dehydrated over $18 \mathrm{~h}$ in

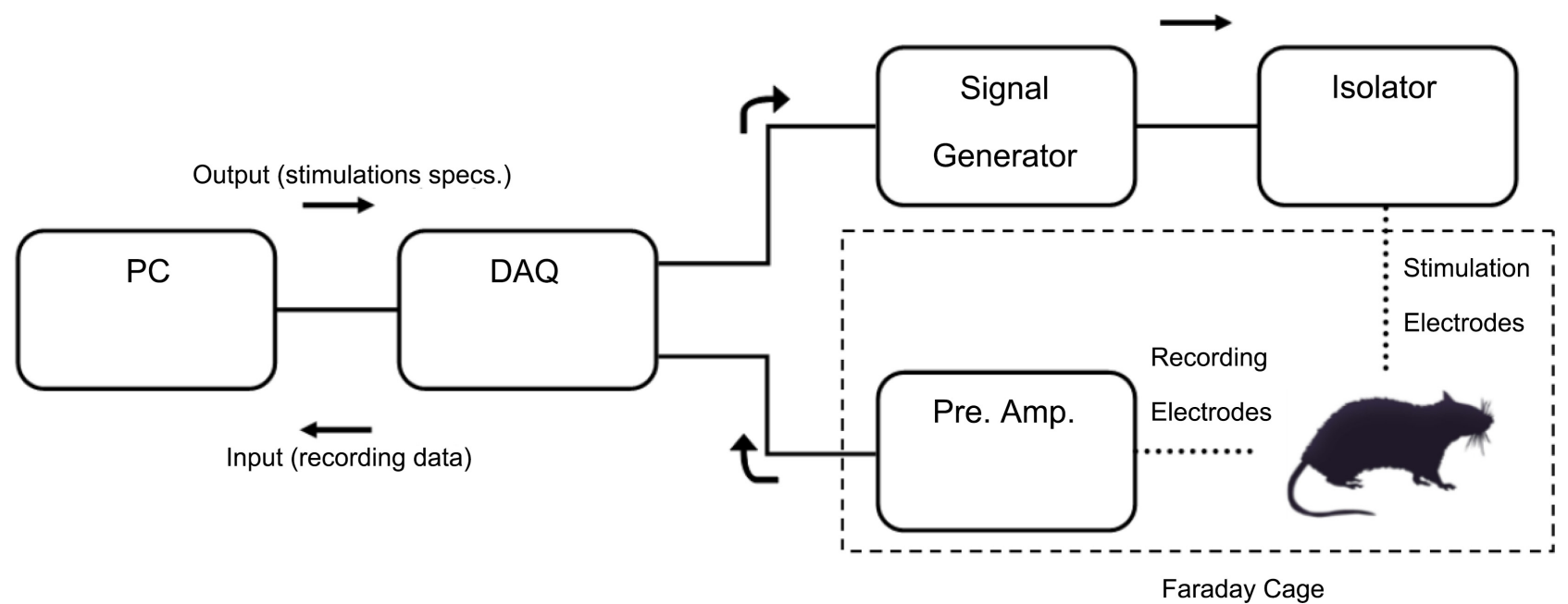

Figure 1. Diagram of electrophysiological experimental circuit. A Data Acquisition unit (DAQ) converts the digital output from a PC to an analogue signal, which triggers a signal generator to produce a stimulus pulse of the desired duration and voltage. This pulse is passed through an isolator, this enables the current to be increased if needed and isolates the recording part of the circuit from the power supply, reducing noise. The stimulus pulse is led through stainless steel electrodes into the experimental animal. Platinum recording electrodes record the resulting stimulus response, which is amplified and filtered through a preamplifier. The DAQ then converts the analogue stimulus response to a digital signal which is stored on the PC. The recordings were carried out inside a Faraday cage. 
three steps in ethanol solutions of 70\%, 96\% and 99\%, respectively, and subsequently cleared with estisol and paraffin infiltration. The nerves were cast in paraffin wax and transactional slices were cut with a thickness of $6 \mu \mathrm{m}$ and spacing of $200-300 \mu \mathrm{m}$. The tissue was stained with luxol-fast blue myelin stain $[29,30]$. Nerve slices were examined using light microscopy (ZEISS, Primo Star Microscope, Germany). Digital images were acquired of each slice using 10× magnification with a ZEISS AxioCam ERc 5S microscope camera. Quantification of the amount of myelin stain was carried out using ImageJ software and the total area of the nerve fiber was determined. When several fibers were visible in a slice, the largest (main) fiber was chosen. Using threshold detection, the area covered with myelin stain in each slice was established. This area was then divided by the total nerve fiber area for each individual slice in order to calculate the relative degree of myelination in each slice.

\subsection{Statistics}

N-way ANOVA was performed using MatLab (v. R2015a) software to determine whether DM or HBOT had significant effect upon the electrophysiological measurements. Results from histological examinations were likewise subjected to non-parametric ANOVA (Kruskal-Wallis test) statistics to determine whether DM or HBOT had effect upon the relative amount of myelin in each nerve. N-way ANOVA was chosen because of uneven sized data pools which resulted from animals having to be euthanized before experiments could begin, due to the effects of STZ administration. Experimental permission was granted by the national council for animal research (\#2014-15-0201-00327).

All data is available https://doi.org/10.6084/m9.figshare.12954827.v2.

\section{RESULTS}

The STZ treated animals were confirmed diabetic with Bayer Contour test strips at STZ+14 days. Average blood sugar levels were $26.87 \pm 2.53 \mathrm{mmol} / \mathrm{L}$, (pre STZ average, $6.23 \pm 0.27 \mathrm{mmol} / \mathrm{L}$ ). The blood sugar levels of nine animals exceeded the detection range of the blood sugar meter $(>33.3 \mathrm{mmol} / \mathrm{L})$. These values at not included in the average mentioned above.

Figure 2(A) shows LEP from two representative animals: one in the HBOT (blue) and one in the non-HBOT (black). LEP amplitude is lowest and latency longest for the animals in the non-HBOT-group. Figure 2(B) displays scatter plots of LEP onset times, while Figure 2(C) shows LEP amplitude.

Figure 3 displays data from MEP recordings from the different groups. Figure 3(A) shows two examples of representative MEPs; blue line is HBOT, while black line is non-HBOT. Figure 3(B) displays scatter plots of conduction speed from the stimulation site across the neuromuscular junction to the muscles. In Figure 3(C), RMS levels of MEPs are presented for the various groups.

Figures 4(A)-(D) show examples of sciatic nerve slices from each experimental group, whilst Figure 4(E) displays scatterplots of the relative myelin area for each group.

DM and HBOT both exerted a statistically significant effect on LEP onset times, $p=0.046$ and $p=$ 0.0003 respectfully. Likewise, analysis of LEP peak-peak amplitudes displayed a statistically significant effect of HBOT $(p=0.033)$, however not for DM. For sciatic nerve conduction speeds, a statistically significant effect was found for DM ( $p=0.0016)$, but not for HBOT, and no statistically significant effect was found on RMS levels of MEPs for neither HBOT nor DM.

ANOVA of histological analyses showed a statistically significant effect of HBOT upon the relative myelin area of the nerve slices from the different groups, the HBOT treated animals showed greater relative myelin area, $(p=0.019)$, while the effect of DM was not statistically significant.

HBOT animals were daily monitored by veterinary staff and showed no indication of oxygen toxicity. All animals which underwent HBOT appeared healthy at the studies end.

\section{DISCUSSION}

The main findings of the experiment were that DM affected LEP onset times and nerve conduction 

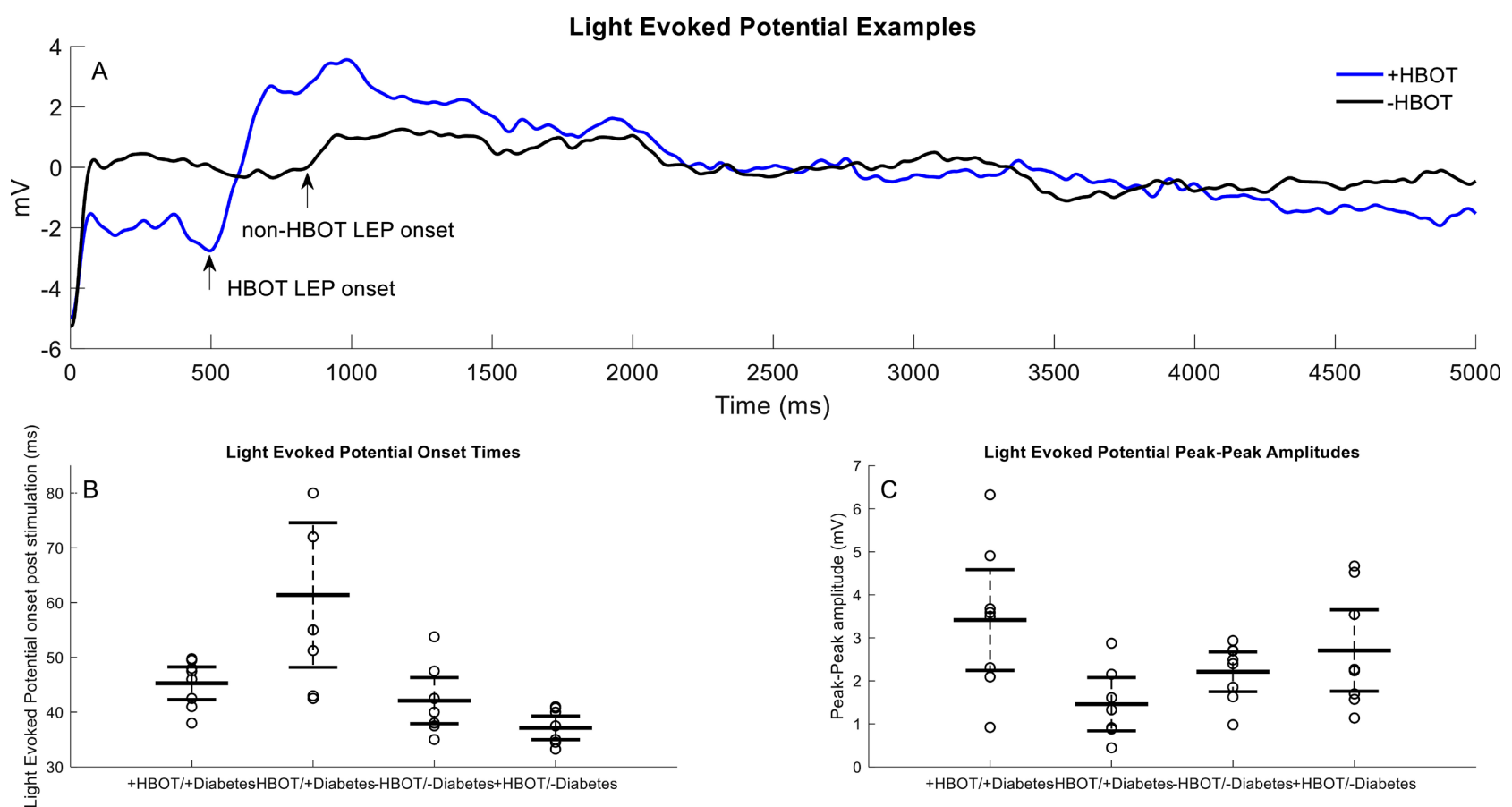

Figure 2. Light evoked potentials (LEPs) data. (A) LEPs from one representative diabetic animal in the Hyperbaric oxygen treatment (HBOT) group and non-HBOT treated group (B) LEP onset time of the experimental groups. (C) Peak-to-peak amplitude of the experimental groups. Horizontal lines indicate means and $95 \%$ confidence intervals.
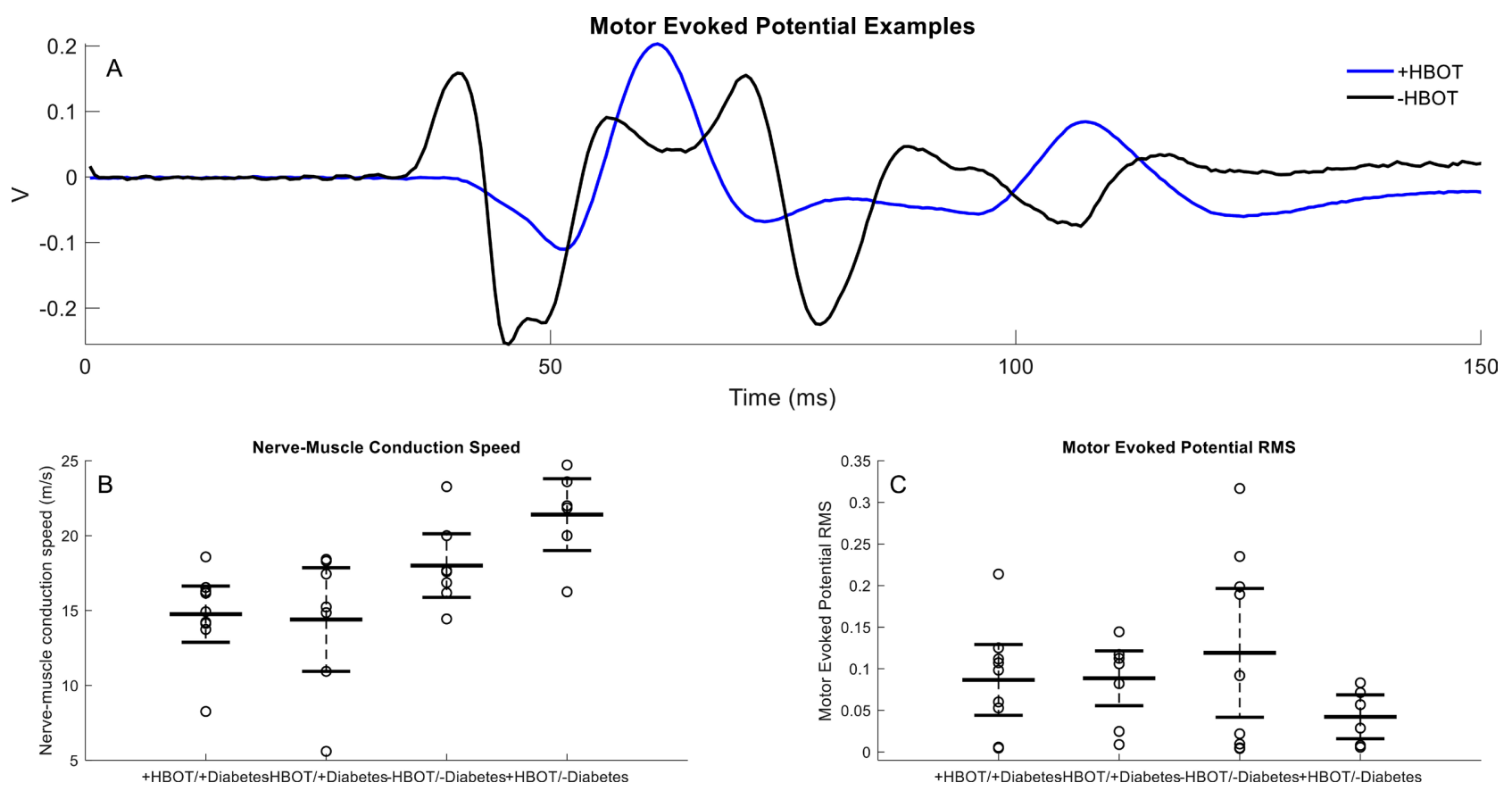

Figure 3. Muscle evoked potential (MEPs) (A) MEPs from one representative diabetic animal in the Hyperbaric oxygen treatment (HBOT) group and non-HBOT treated group (B) Conduction speed along sciatic nerve across to muscle for experimental groups (C) Root mean square (RMS) of the experimental groups. Horizontal lines indicate means and $95 \%$ confidence intervals. 

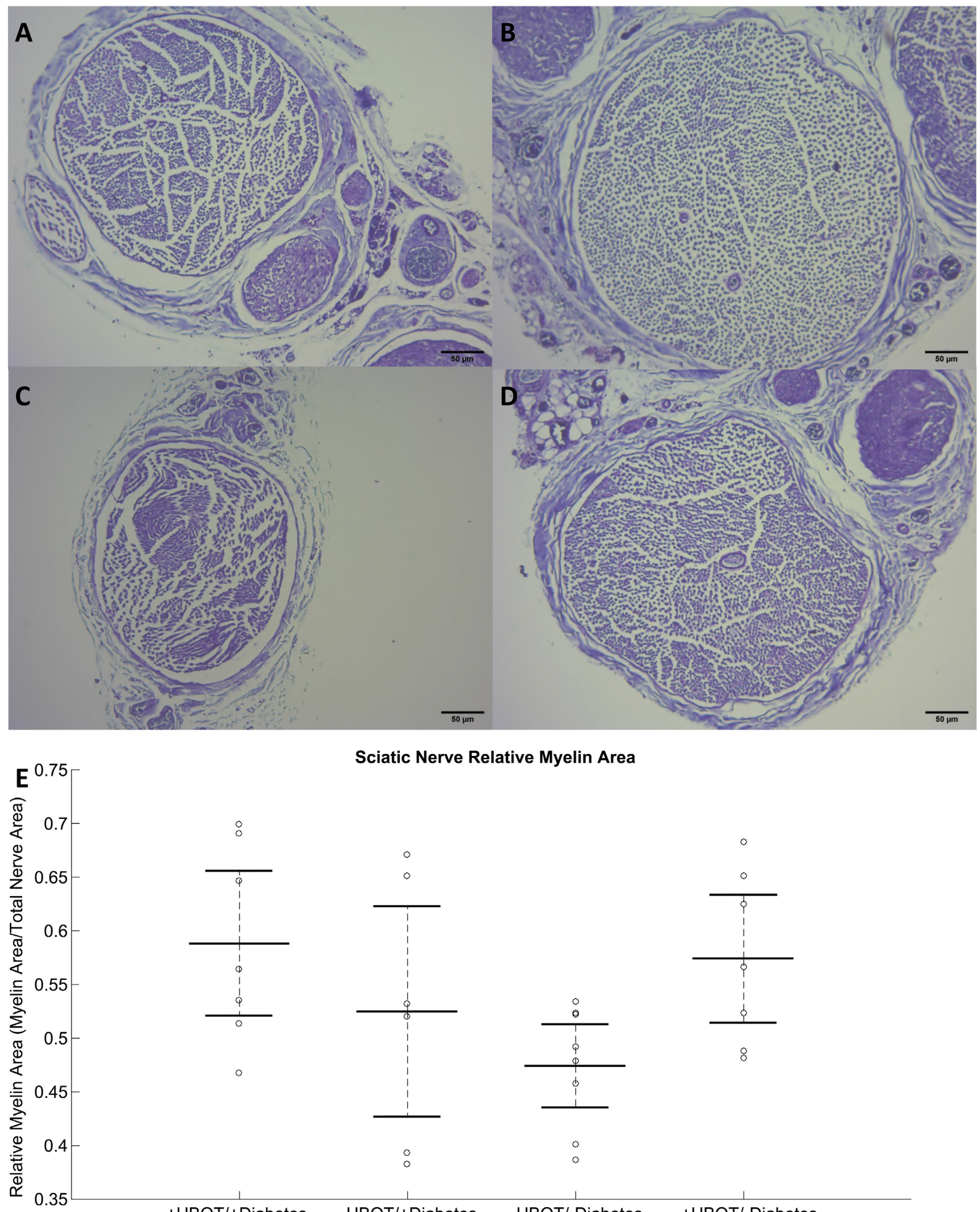

Sciatic Nerve Relative Myelin Area 
speeds (Figure 2 and Figure 3). Furthermore, both LEP onset times and amplitudes improved significantly in HBOT animals, however, neither conduction speed nor MEP RMS were changed following HBOT. For LEP onset times, the groups displaying the largest latency were the diabetic groups and the best performing group was the healthy controls receiving HBOT. Likewise, the groups with the highest LEP amplitudes were the HBOT-groups, both control and diabetic. These results therefore indicate a positive effect upon retina in diabetic and control groups. The positive effect of HBOT on all animal LEP measurements might be due to the inherently high energy turnover of the mammalian retina. Since the retina is embryologically a projection of the forebrain [31], it is a part of the CNS and consequently has a very high energy demand $[32,33]$. Therefore, the large availability of oxygen during HBOT may enhance the performance of the retina, even in healthy controls.

It is important to note that electrophysiological experiments were carried out one week after the final HBOT session, thus seven days where the animals had been breathing normal atmospheric air: therefore, the observed effects of HBOT cannot be caused by potential effects of elevated dissolved oxygen per se at the time of recording.

In the peripheral nerves, only DM had a statistically significant effect, and only on conduction speeds, not RMS levels. Differences in vascularization compared with the retina may explain the lack of effect of HBOT in peripheral nerves. The high energy demand mentioned and the need to remove heat conducted by light to the retina necessitates high blood perfusion to the retina. Therefore, the large increase in dissolved oxygen, caused by HBOT, can most likely reach the entire retina in relatively short time, which is in contrast to peripheral nerves, where no special vascular structures exist. As such, the relative short burst of elevated oxygen may be of too short duration to deliver sufficient oxygen necessary to improve peripheral nerve function, even though animals of the sizes used in this study should become saturated relatively quickly (well within the intervention period) [34].

The histological analyses showed increased myelin in HBOT animals, and that DM had no significant effect on myelination. As mentioned, the only statistically significant effect on the peripheral nerves, regarding electrophysiology, was caused by DM. The degree of myelination in untreated nerves, both in healthy and diabetic animals, was sufficient to maintain conduction speeds because the nerves of HBOT-animals did not display higher conduction speeds compared to those who were not subjected to HBOT. Also, the lack of any effect of DM in the histological evaluation suggested that whatever electrophysiological effects caused by DM in the peripheral nerves, it was either: not visible at the sites of histological examination, but perhaps present at a more distal point of the nerves, and/or caused at the molecular level. As the development time for DR and DN using the induction method described here is not known whether the effects observed were strictly metabolic or in part caused by tissue damage/changes caused by DM.

Our findings support previous studies about the effect of HBOT on DR and DN. One study reported positive effect of HBOT on the breakdown of the blood-retina barrier found in DR [35] and this reduced breakdown could explain the improved retinal function in HBOT treated animals. Similar to our study, the authors also used an STZ diabetic rat model, but applied a different HBOT protocol (2.5 atm of 100\% $\mathrm{O}_{2}$ for $90 \mathrm{~min}$, Monday, Wednesday and Fridays for 3 months). Therefore, a more significant effect of HBOT could possibly have been found if a more intense HBOT regime had been used, as in the present study. Other studies have shown positive but passing effect of HBOT upon DR [36]. In light of this, we found it interesting that our study showed positive effect of HBOT 7 days post HBOT cessation.

Our finding of no effect of HBOT on conduction speed of the motor neurons is in line with the findings by Low et al. [22], who further reported improved nerve evoked potential amplitudes, however, utilizing a different measure for amplitude in that recordings were carried out in the connecting muscles and not in the nerves themselves. When recording in muscles, MEP RMS levels are indirect measures of the ability of the motor neuron to produce action potentials [37]. Our choice of measuring in muscle was based on expedience as measurement of MEPs from muscles is a relatively simple and robust procedure. Likewise, our approach would allow measurements of motor nerves to the most distal parts of the motor nerves, increasing the likelihood of detecting neuropathic abnormalities. 
The measurements used to study the effects of DM in peripheral nerves (conduction speed and MEP RMS) were chosen because these measures can detect well-known consequences of DN; 1) demyelination, which causes slowing of action potentials [38] and decreased amplitude of the compound action potential due to dispersion of the individual action potentials; and 2) axonal loss, which also leads to decreased amplitude of compound action potential, a reduced release of acetylcholine in the neuromuscular junction, and reduced MEP [39].

An important consideration regarding HBOT is hyperoxia-induced vasoconstriction. The causes of hyperoxia vasoconstriction are multifaceted, including: 1) reduction of nitric oxide radicals, leading to decreased vasorelaxant effect; 2) alteration of vasodilator compounds causing net vasoconstriction [40]; and 3 ) increased sympathetic tone from the CNS resulting in vasoconstriction [41]. Vasoconstriction is therefore a well-documented consequence of hyperoxia. Vasoconstriction will potentially counteract effective oxygen delivery during HBOT if the effect is not outweighed by the increased dissolved blood oxygen delivery. As such, there could exist an equilibrium between these opposing forces, where the effects will (dependent upon oxygen tension) become either positive $[42,43]$ or deleterious [44] to nervous and retinal performance. This mechanism may also explain the difference in the electrophysiological results in the peripheral nerves and eyes. Depending on the degree of vascularization and possible differing vasoconstriction, there may be positive effect of HBOT in one tissue type, and no effect in another.

A further consideration regarding HBOT as a feasible treatment for DR and DN is oxygen toxicity. Oxygen toxicity causes harmful effects in various tissues, including the CNS, lungs and eyes [45]. These detrimental effects put a severe restriction upon the use of HBOT and the duration of treatment. Furthermore, the act of increasing and decreasing blood oxygen tension during HBOT sessions likely causes a myriad of secondary responses and processes (hyperoxic conditioning), which if used in humans, needs to be understood and examined for possible detrimental effects.

\section{CONCLUSION}

In conclusion, we found a beneficial effect of HBOT on DR as well as increased myelination in peripheral nerves of rats following STZ induced DM. No significant beneficial effect of HBOT was found on peripheral nerve conduction. Future studies should combine DM animal models and long-term HBOT regimens.

\section{CONFLICTS OF INTEREST}

The authors declare no conflicts of interest regarding the publication of this paper.

\section{REFERENCES}

1. WHO (2016) Global Report Diabetes. World Health Organization, Geneva.

2. EDPRG (2004) The Prevalence of Diabetic Retinopathy among Adults in the United States. Archives of Ophthalmology, 122, 552-563. https://doi.org/10.1001/archopht.122.4.552

3. Maser, R.E., Steenkiste, A.R., Dorman, J.S., Nielsen, V.K., Bass, E.B., Manjoo, Q., et al. (1989) Epidemiological Correlates of Diabetic Neuropathy. Diabetes, 38, 1456-1461. https://doi.org/10.2337/diabetes.38.11.1456

4. Tesfaye, S. and Selvarajah, D. (2012) Advances in the Epidemiology, Pathogenesis and Management of Diabetic Peripheral Neuropathy. Diabetes/Metabolism Research and Reviews, 28, 8-14.

https://doi.org/10.1002/dmrr.2239

5. Geoffrey, B.A. and Sobha, S. (2011) Hypoxia and Oxidative Stress in the Causation of Diabetic Retinopathy. Current Diabetes Review, 7, 291-304. https://doi.org/10.2174/157339911797415620

6. Dyck, P.J., Lais, A., Karnes, J.L.K., O’Brien, P. and Rizza, R. (1986) Fiber Loss Is Primary and Multifocal in Sural Nerves in Diabetic Polyneuropathy. Annals of Neurology, 19, 425-439. https://doi.org/10.1002/ana.410190503 
7. Dyck, P.J., Karnes, J.L.K., O’Brien, P., Okazaki, H., Lais, A. and Engelstad, J. (1986) The Spatial Distribution of Fiber Loss in Diabetic Polyneuropathy Suggests Ischemia. Annals of Neurology, 19, 440-449. https://doi.org/10.1002/ana.410190504

8. Goncalves, N.P., Vægter, C.B. andersen, H., Østergaard, L., Calcutt, N.A. and Jensen, T.S. (2017) Schwann Cell Interactions with Axons and Microvessels in Diabetic Neuropathy. Nature Reviews Neurology, 13, 135-147. https://doi.org/10.1038/nrneurol.2016.201

9. Williams, E., Timperley, W.R., Ward, J.D. and Duckworth, T. (1980) Electron Microscopical Studies of Vessels in Diabetic Peripheral Neuropathy. Journal of Clinical Pathology, 33, 462-470. https://doi.org/10.1136/jcp.33.5.462

10. Gianninin, C. and Dyck, P.J. (1995) Basement Membrane Reduplication and Pericyte Degeneration Precede Development of Diabetic Polyneuropathy and Are Associated with Its Severity. Annals of Neurology, 37, 498-504. https://doi.org/10.1002/ana.410370412

11. Hill, R.E. and Williams, P.E. (2004) Perineurial Cell Basement Membrane Thickening and Myelinated Nerve Fibre Loss in Diabetic and Nondiabetic Peripheral Nerve. Journal of Neurological Sciences, 217, 157-163. https://doi.org/10.1016/j.jns.2003.09.011

12. Patz, A. (1980) Studies on Retinal Neovasculariztion. Investigative Ophthalmology \& Visual Science, 19, 1133-1138.

13. Rand, L.I. (1981) Recent Advances in Diabetic Retinopathy. The American Journal of Medicine, 70, 595-602. https://doi.org/10.1016/0002-9343(81)90581-7

14. Gong, C.Y., Lu, B., Hu, Q.W. and Ji, L.L. (2013) Streptozotocin Induced Diabetic Retinopathy in Rat and the Expression of Vascular Endothelial Growth Factor and Its Receptor. International Journal of Ophthalmology, 6, 573-577.

15. Kovacs, B., Lumayag, S., Cowan, C. and Xu, S. (2011) MicroRNAs in Early Diabetic Retinopathy in Streptozotocin-Induced Diabetic Rats. Investigative Ophthalmology \& Visual Science, 52, 4402-4409. https://doi.org/10.1167/iovs.10-6879

16. Lai, A.K.W. and Lo, A.C.Y. (2013) Animal Models of Diabetic Retinopathy, Summary and Comparison. Journal of Diabetes Research, 2013, Article ID: 106594. https://doi.org/10.1155/2013/106594

17. Bravenboer, B., Kappelle, A.C., Hamers, F.P.T., Buren, T., Erkelens, D.W. and Gispen, W.H. (1992) Potential Use of Glutathione for the Prevention and Treatment of Diabetic Neuropathy in the Streptozotocin-Induced Diabetic Rat. Diabetologia, 35, 813-817. https://doi.org/10.1007/BF00399926

18. Fox, A., Eastwood, C., Gentry, C., Manning, D. and Urban, L. (1999) Critical Evaluation of the Streptozotocin Model of Painful Diabetic Neuropathy in the Rat. Pain, 81, 307-316. https://doi.org/10.1016/S0304-3959(99)00024-X

19. Greene, D.A., De Jesus, P.V. and Winegrad, A.I. (1975) Effects of Insulin and Dietary Myoinositol on Impaired Peripheral Motor Nerve Conduction Velocity in Acute Streptozotocin Diabetes. Journal of Clinical Investigation, 55, 1326-1336. https://doi.org/10.1172/JCI108052

20. Löndahl, M., Katzman, P., Nilsson, A. and Hammarlund, C. (2010) Hyperbaric Oxygen Therapy Facilitates Healing of Chronic Foot Ulcers in Patients with Diabetes. Diabetes Care, 33, 998-1003. https://doi.org/10.1172/JCI108052

21. Abidia, A., Laden, G., Kuhan, G., Johnson, B.F., Wilkins, A.R., Renwick, P.M., et al. (2003) The Role of Hyperbaric Oxygen Therapy in Ischaemic Diabetic Lower Extremity Ulcers, a Double-Blind Randomised-Controlled Trial. European Journal of Vascular and Endovascular Surgery, 25, 513-518. https://doi.org/10.1053/ejvs.2002.1911

22. Low, P.A., Schmelzer, J.D., Ward, K.K., Curran, G.L. and Poduslo, J.F. (1988) Effect of Hyperbaric Oxygenation 
on Normal and Chronic Streptozotocin Diabetic Peripheral Nerves. Experimental Neurology, 99, 201-212. https://doi.org/10.1016/0014-4886(88)90139-2

23. Bresnick, G.H. and Palta, M. (1987) Temporal Aspects of the Electroretinogram in Diabetic Retinopathy. Archives of Ophthalmology, 105, 660-664. https://doi.org/10.1001/archopht.1987.01060050078042

24. Seidl, R., Birnbarcher, R., Hauser, E., Bernert, G., Freilinger, M. and Schober, E. (1996) Brainstem Auditory Evoked Potentials and Visually Evoked Potentials in Young Patients with IDDM. Diabetes Care, 19, 1220-1224. https://doi.org/10.2337/diacare.19.11.1220

25. Tesch, G.H. and Allen, T.J. (2007) Rodent Models of Streptozotocin-Induced Diabetic Nephropathy. Nephrolo$g y, 12,261-266$. https://doi.org/10.1111/j.1440-1797.2007.00796.x

26. Coppery, L.J., Davidson, E.P., Dunlap, J.A., Lund, D.D. and Yorek, M.A. (2000) Slowing of Motor Nerve Conduction Velocity in Streptozotocin Induced Diabetic Rats Is Preceeded by Impaired Vasodialation in Arteriols That Overlie the Sciatic Nerve. International Journal of Experimental Diabetes Research, 1, 131-143. https://doi.org/10.1155/EDR.2000.131

27. Goss-Sampson, M.A. and Kriss, A. (1991) Effects of Pentobarbital and Ketamine-Xylazine Anesthesia on Somatosensory, Brainstem Auditory and Peripheral Sensory-Motor Responses in the Rat. Laboratory Animals, 25, 360-366. https://doi.org/10.1258/002367791780810074

28. Nørlinger, T.S., Nielsen, P.M., Qi, H., Mikkelsen, E., Hansen, K., Schmidt, N.H., et al. (2017) Hyperbaric Oxygen Therapy Reduces Renal Lactate Production. Physiological Reports, 5, e13217.

https://doi.org/10.14814/phy2.13217

29. Shi, X., Chen, Y., Nadeem, L. and Xu, G. (2013) Beneficial Effect of TNF- $\alpha$ Inhibition on Diabetic Peripheral Neuropathy. Journal of Neuroinflammation, 10, 283-288. https://doi.org/10.1186/1742-2094-10-69

30. Wang, I.C., Chung, C.Y., Liao, F., Chen, C.C. and Lee, C.H. (2017) Peripheral Sensory Neuron Injury Contributes to Neuropathic Pain in Experimental Autoimmune Encephalomyelitis. Scientific Reports, 7, Article No. 42304. https://doi.org/10.1038/srep42304

31. Chuang, J.C. and Raymond, P.A. (2001) Zebrafish Genes rx1 and rx2 Help Define the Region of Forebrain That Gives Rise to Retina. Developmental Biology, 231, 13-30. https://doi.org/10.1006/dbio.2000.0125

32. Ames, A. (1992) Energy Requirements of CNS Cells as Related to Their Function and to Their Vulnerability to Ischemia, a Commentary Based on Studies on Retina. Canadian Journal of Physiology and Pharmacology, 70, S158-S164. https://doi.org/10.1139/y92-257

33. Wong-Riley, M.T. (2010) Energy Metabolism of the Visual System. Eye Brain, 2, 99. https://doi.org/10.2147/EB.S9078

34. Fahlman, A. (2017) Allometric Scaling of Decompression Sickness Risk in Terrestrial Mammals, Cardiac Output Explains Risk of Decompression Sickness. Scientific Reports, 7, Article No. 40918.

https://doi.org/10.1038/srep40918

35. Chang, Y.H., Chen, P.L., Tai, M.C., et al. (2006) Hyperbaric Oxygen Therapy Ameliorates the Blood-Retinal Barrier Breakdown in Diabetic Retinopathy. Clinical \& Experimental Ophthalmology, 34, 584-589. https://doi.org/10.1111/j.1442-9071.2006.01280.x

36. Butler, F.K., Hagan, C. and Murphy-Lavoie, H. (2008) Hyperbaric Oxygen Therapy and the Eye. Undersea \& Hyperbaric Medicine, Journal of the Undersea and Hyperbaric Medical Society, Inc., 35, 333-387.

37. Raff, M.C. and Asbury, A.K. (1968) Ischemic Mononeuropathy and Mononeuropathy Multiplex in Diabetes Mellitus. The New England Journal of Medicine, 279, 17-21. https://doi.org/10.1056/NEJM196807042790104

38. Thomas, P.K. and Lascelles, R.G. (1966) The Pathology of Diabetic Neuropathy. International Journal of Medicine, 35, 489-509. 
39. Wilson, J.R., Stittsworth, J.D., Kadir, A. and Fisher, M.A. (1999) Conduction Velocity versus Amplitude Analysis, Evidence for Demyelination in Diabetic Neuropathy. Muscle \& Nerve, 21, 1228-1230. https://doi.org/10.1002/(SICI)1097-4598(199809)21:9<1228::AID-MUS20>3.0.CO;2-M

40. Goyal, A., Chonis, T. and Cooper, J.S. (2020) Hyperbaric Cardiovascular Effects. StatPearls, Treasure Island.

41. Gaiser, H.G., Demchenko, I.T., Zhilyaev, S.Y., Moskvin, A.N., Krivchenko, A.I. and Piantadosi, C.A. (2018) Adrenoceptor Blockade Modifies Regional Cerebral Blood Flow Responses to Hyperbaric Hyperoxia, Protection against CNS Oxygen Toxicity. Journal of Applied Physiology, 125, 1296-1304. https://doi.org/10.1152/japplphysiol.00540.2018

42. Jansen, E.C. and Nielsen, N.V. (2004) Promising Visual Improvement of Cystoid Macular Oedema by Hyperbaric Oxygen Therapy. Acta Opthalmologica Scandinavica, 82, 485-486. https://doi.org/10.1111/j.1395-3907.2004.00305.x

43. Maalej, A., Khallouli, A., Choura, R., Ben Sassi, R., Rannen, R. and Gharsallah, H. (2020) The Effects of Hyperbaric Oxygen Therapy on Diabetic Retinopathy, A Preliminary Study. Journal Français d̛ Ophtalmologie, 43, 133-138. https://doi.org/10.1016/j.jfo.2019.07.005

44. Tran, V. and Smart, D. (2017) Proliferative Retinopathy during Hyperbaric Oxygen Treatment. Diving and Hyperbaric Medicine, 47, 203. https://doi.org/10.28920/dhm47.3.203

45. Chawla, A. and Lavania, A.K. (2001) Oxygen Toxicity. Medical Journal Armed Forces India, 57, 133-137. https://doi.org/10.1016/S0377-1237(01)80133-7 